Brit. J. Ophthal. (1958) 42, 680.

\title{
CHANGES IN OCULAR RIGIDITY IN ENDOCRINE EXOPHTHALMOS*
}

\author{
BY \\ R. WEEKERS AND G. LAVERGNE \\ From the Ophthalmological Clinic, Liége University
}

Two types of endocrine exophthalmos are frequently distinguished, being $\frac{\mathscr{O}}{0}$. referred to thyrotoxic or hyperthyroid .exophthalmos, and thyrotropic, ophthalmoplegic, or oedematous exophthalmos.

(a) Hyperthyroid or Thyrotoxic Exophthalmos.-This accompanies Graves's disease and is, therefore, much more frequently seen in females than in males. In the majority of cases the exophthalmos is quite unobtrusive or there is merely an appearance of exophthalmos due to retraction of the upper lid. It is associated with a decreased frequency of blinking and a fixed stare. The majority of authors agree that these symptoms should be attributed to an increase in tone of the sympathetic system. The importance of the pituitary thyrotropic hormone in this clinical picture is not clear. Hyperthyroid exophthalmos is not complicate either by chemosis or by diplopia, but heterophoria and lack of convergence are often seen.

(b) Thyrotropic, Ophthalmoplegic, or Oedematous Exophthalmos.-This may occur either in a subject suffering from verified and treated hyperthyroidism, when the signs of thyrotoxicosis are about to disappear, or in an apparently normal subject free from any thyroid symptom or history of symptoms. The second type is more frequently seen in males than in females.

Thyrotropic exophthalmos is often very marked 'and may even lead to irreducible lagophthalmos; it is invariably associated with a disturbance of ocular movements, particularly with elevation of the gaze. More or less marked chemosis sometimes complicates this condition. It is believed to be caused by the secretion of an exophthalmos-producing substance directly related to the thyrotropic hormone.

These two clinical entities, thyrotoxic and thyrotropic exophthalmos, may be encountered in the same subject. When a goitre is given treatment, a typical hyperthyroid type of exophthalmos is often seen to become more pronounced and to acquire the features of thyrotropic exophthalmos. Such transitions are so frequent that cases of either purely hyperthyroid or purely thyrotropic exophthalmos are relatively rare.

Albert (1945) discovered that exophthalmos could be induced in minnows by the injection of anterior pituitary extract. On the other hand, Dobyns

* Received for publication January 14, 1958. 
and Wilson (1954) demonstrated that injection of serum from certain exophthalmic patients gives rise to ocular protrusion in the goldfish Carassius auratus (Japanese variety), whereas serum from other patients produced no such effect. This new test would seem to throw some light on the pathogenesis of endocrine exophthalmos.

This paper reports an attempt to reach an analogous goal using a different method. In a recent paper (Weekers and Lavergne, 1957), we have demonstrated that ocular rigidity is considerably reduced in thyrotropic exophthalmos but remains normal in thyrotoxic exophthalmos. Our observations at present cover a series of 26 cases of thyrotoxic and six cases of thyrotropic exophthalmos. Three of the latter cases were made available by Prof. J. François of Ghent, whose co-operation in this respect we gratefully acknowledge.

The techniques of determining the coefficient of rigidity $(K)$ need not be described here, as they have been the subject of many previous publications (Friedenwald, 1937, 1954, 1957; Schmidt, 1956; Goldmann and Schmidt, 1957; Lavergne, Prijot, and Weekers, 1957).

The average ocular rigidity in normal subjects usually varies between 0.0200 and 0.0250 , and it shows a somewhat wide margin of fluctuation in normal conditions, even in the absence of any anomaly of the eyeball (Table I).

TABLE I

FLUCTUATIONS IN OCULAR RIGIDITY $(K)$ IN NORMAL SUBJECTS

\begin{tabular}{|c|c|c|c|c|c|c|}
\hline \multicolumn{3}{|c|}{ Author } & \multirow{2}{*}{$\begin{array}{l}\text { Date } \\
1937\end{array}$} & \multirow{2}{*}{$\begin{array}{c}\begin{array}{c}\text { Number of } \\
\text { Cases }\end{array} \\
500\end{array}$} & \multirow{2}{*}{$\frac{\begin{array}{c}\text { Average } K \\
\text { (Ocular Rigidity) }\end{array}}{0.0215}$} & \multirow{2}{*}{$\begin{array}{c}\begin{array}{c}\text { Physiological } \\
\text { Variation }\end{array} \\
0.0060 \text { to } 0.0370\end{array}$} \\
\hline Friedenwald & . & .. & & & & \\
\hline Schmidt & .. & .. & 1956 & - & 0.0225 & $s=0.0089$ \\
\hline Personal Obse & & .. & 1957 & 239 & 0.0246 & $s=0.0063$ \\
\hline
\end{tabular}

Table $\Pi$ shows that in hyperthyroid subjects the ocular rigidity either remains normal or shows only very slight changes as long as the ocular affection retains the features of thyrotoxic exophthalmos as described, i.e. as long as there is no change in ocular motility and no chemosis.

TABLE II

FLUCTATIONS IN OCULAR RIGIDITY IN 26 CASES OF HYPERTHYROID EXOPHTHALMOS

\begin{tabular}{|c|c|c|c|c|}
\hline \multirow{2}{*}{\multicolumn{2}{|c|}{ Number of Eyes }} & \multicolumn{3}{|c|}{$K$ (Ocular Rigidity) } \\
\hline & & Minimum & Average & Maximum \\
\hline $52 \ldots$ & .. & 0.0170 & 0.0226 & 0.0325 \\
\hline
\end{tabular}


Table III, on the other hand, shows a marked decrease in ocular rigidity in six cases of thyrotropic exophthalmos with a change in ocular motility and chemosis.

TABLE III

DECREASE IN OCULAR RIGIDITY IN SIX PATIENTS WITH THYROTROPIC EXOPHTHALMOS

\begin{tabular}{|c|c|c|c|c|c|}
\hline \multirow{2}{*}{\multicolumn{3}{|c|}{ Number of Eyes }} & \multicolumn{3}{|c|}{$K$ (Ocular Rigidity) } \\
\hline & & & Minimum & Average & Maximum \\
\hline $12 \ldots$ & $\ldots \quad \ldots$ & $\ldots \quad \ldots$ & 0.0080 & 0.0115 & 0.0150 \\
\hline
\end{tabular}

Four of these patients were hyperthyroids, and were being treated either with radioactive iodine or with antithyroid substances. The other two patients showed thyrotropic exophthalmos without goitre or Graves's disease, and were receiving no treatment.

Chemosis seems to be the clinical symptom which heralds a decrease in ocular rigidity. This was normal in three cases of bilateral ophthalmoplegia of endocrine origin, which showed similarities to thyrotropic exophthalmos but lacked conjunctival oedema.

Apart from its value as an aid in diagnosing the nature of an endocrine exophthalmos, ocular rigidity is also of importance in the determination of the ocular tension. Endocrine exophthalmos-and particularly, or so it would seem to us, thyrotropic exophthalmos-is sometimes complicated by a pathological increase in ocular tension. The calibration tables of the Schiötz tonometer have been calculated on the basis of a rigidity of 0.0215. Tonometric determinations show certain errors when ocular rigidity is abnormally low. As a result, ocular hypertension is only too readily overlooked or underestimated in subjects with thyrotropic exophthalmos in whom ocular rigidity is decreased (Table IV, opposite).

Table IV shows the frequency of ocular hypertension in cases of thyrotropic exophthalmos. On the other hand, the ocular tension was normal in all cases of thyrotoxic exophthalmos included in this investigation.

The hypertension seen in thyrotropic exophthalmos shows varying degrees of intensity. In the majority of cases it is moderately marked and not associated with any change in the irido-corneal angle. In that case it develops parallel with the exophthalmos and recedes with its regression within a few months or years. It is favourably influenced by therapeutic measures which reduce the aqueous flow (Diamox or 2 per cent. adrenaline), but it improves only very slightly after treatment with miotics. However unobtrusive, it may give rise to glaucomatous defects in the field of vision (Figure, opposite). 
TABLE IV

CORRECTION OF TONOMETRIC FINDINGS IN SIX SUBJECTS WITH THYROTROPIC EXOPHTHALMOS AND DECREASED OCULAR RIGIDITY

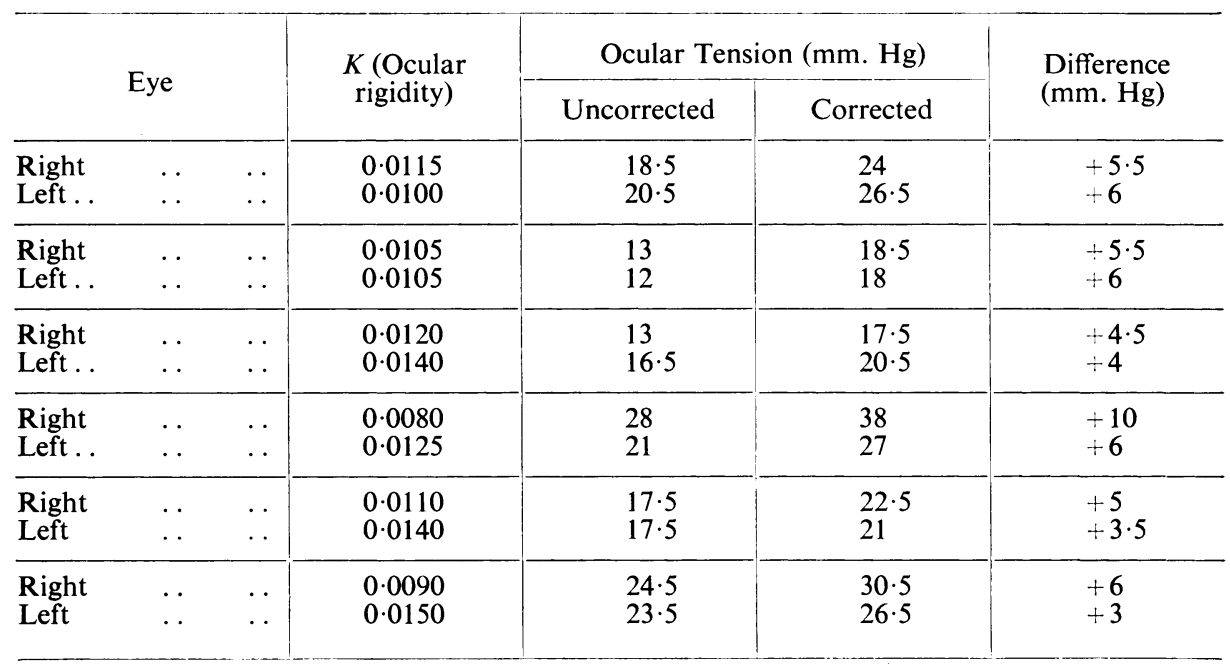

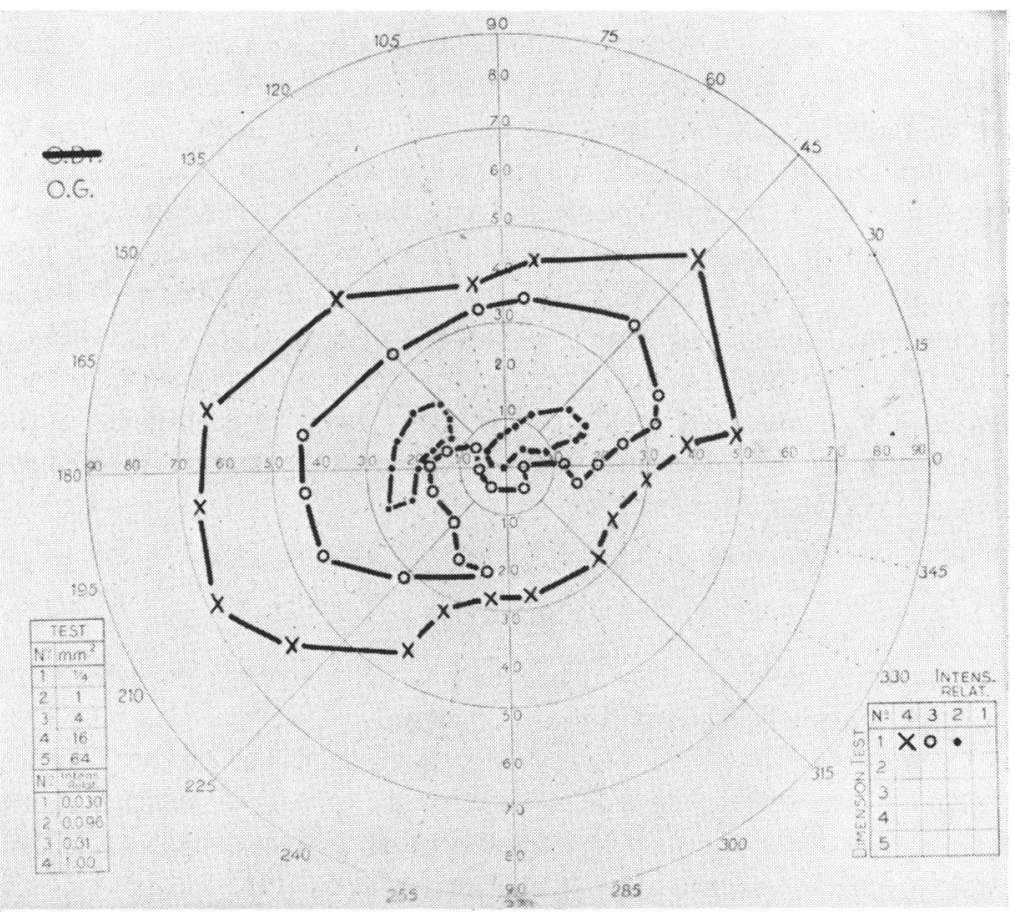

Figure.-Glaucomatous defects in thyrotropic exophthalmos complicated by ocular hypertension (left eye). 
In rare cases hypertension may be manifested by an acute crisis, during which gonioscopy reveals closure of the irido-corneal angle. In such cases any medical treatment is ineffective, and an operation is required. In one of our cases the ocular tension did not become normal until one eye had been submitted to iridencleisis and the other to iridencleisis and angiodiathermy.

\section{Comment}

Thyrotropic exophthalmos is characterized by an increase in the water concentration of various tissues (Wybar, 1957). This phenomenon is seen in the orbit, the extrinsic muscles, and the subconjunctival spaces. It seems that the decrease in ocular rigidity revealed by differential tonometry is attributable to a change in the physical properties of the sclera, which results in an anomalous imbibition. To this phenomenon we may also attribute the abrupt changes in ocular refraction observed in one of our cases. Such changes in cases of exophthalmos with low ocular rigidity merit further investigation.

The pathogenesis of ocular hypertension in thyrotropic exophthalmos has so far remained obscure. It is known that any lasting increase is caused by an increased resistance to the outflow of aqueous humour, an increased flow, or an increase in venous episcleral pressure at the junction of the aqueous veins and blood vessels. The last mechanism can be excluded in the case under discussion, but it is more difficult to determine the relative importance of the increase in resistance and the increase in flow. An increase in resistance to the outflow of aqueous humour is by far the most common mechanism in clinical cases of hypertension. It may be that the imbibition involves the exit-channels from the anterior segment, in which it would create an anomalous resistance. This hypothesis seems highly likely in cases in which gonioscopy shows closure of the irido-corneal angle. The resistance to outflow, is, however, difficult to determine, since the alteration in ocular rigidity makes the exact interpretation of tonographic curves impossible. Moreover, it has not hitherto been possible to make exact determinations of the aqueous flow in cases of hypertension complicating thyrotropic exophthalmos.

\section{Summary}

Ocular rigidity is markedly decreased in thyrotropic exophthalmos, but in thyrotoxic exophthalmos it remains normal.

The change in rigidity in the first case, which seems to be due to anomalous imbibition by the sclera, entails a downward error in normal tonometric results. As a result, ocular hypertension is often overlooked or underestimated in patients suffering from thyrotropic exophthalmos.

The mechanism of ocular hypertension in thyrotropic exophthalmos remains obscure and merits further investigation. 


\section{REFERENCES}

Albert, A. (1945). Endocrinology, 37, 389.

DobYNS, B. M., and WiLson, L. A. (1954). J. clin. Endocr., 14, 1393.

Friedenwald, J. S. (1937). Amer. J. Ophthal., $20,985$.

(1954). Trans. Amer. Acad. Ophthal. Otolaryng., 58, 924.

(1957). Ibid., 61, 108.

GoldmanN, H., and SCHMIDT, T. (1957). Ophthalmologica (Basel), 133, 330.

LAVERGNe, G., PrIJOT, E., and WeEkers, R. (1957). Arch. Ophtal., 17, 256.

SCHMIDT, T. (1956). Klin. Mbl. Augenheilk., 129, 196.

WeEKers, R., and LaVerGNe, G. (1957). Ophthalmologica (Basel), 134, 276.

WyBAR, K. (1957). Fortschr. Augenheilk., 7, 119. 